Document downloaded from:

http://hdl.handle.net/10251/65375

This paper must be cited as:

Martínez Bauset, J.; Guntupalli, L.; Li, F. (2015). Performance Analysis of Synchronous Duty-Cycled MAC Protocols. IEEE Wireless Communications Letters. 4(5):469-472. doi:10.1109/LWC.2015.2439267.

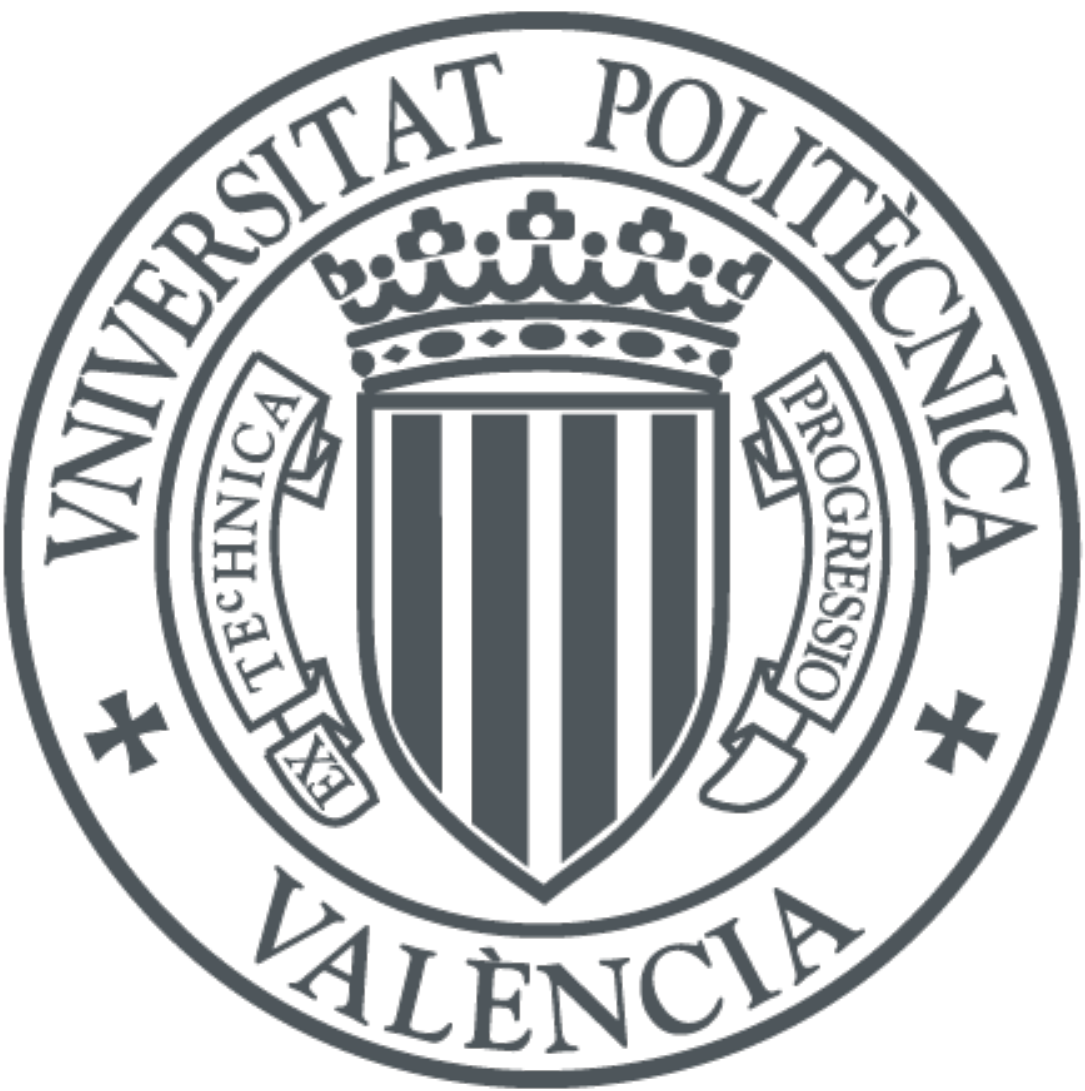

The final publication is available at

http://dx.doi.org/10.1109/10.1109/LWC.2015.2439267

Copyright Institute of Electrical and Electronics Engineers (IEEE)

Additional Information 


\title{
Performance Analysis of Synchronous Duty-Cycled MAC Protocols
}

\author{
Jorge Martinez-Bauset, Lakshmikanth Guntupalli, and Frank Y. Li, Senior Member, IEEE
}

\begin{abstract}
In this letter, we propose an analytical model to evaluate the performance of the S-MAC protocol. The proposed model improves the accuracy of previous models in two aspects. First, it incorporates the dependence among the nodes within a cluster by defining a DTMC that models the number of active nodes, whereas the previous models considered that nodes were mutually independent. Second, it proposes new methods for calculating packet delay and energy consumption. The analytical model is validated through discrete-event based simulations. $\mathrm{Nu}$ merical results demonstrate that the proposed analytical model and methods yield accurate results under realistic assumptions.
\end{abstract}

Index Terms-WSNs, duty-cycled MAC protocols, Markov modeling, delay and energy consumption.

\section{INTRODUCTION}

D UTY cycling (DC) appears as a promising solution to reduce energy consumption in wireless sensor networks (WSNs). In the literature, many medium access control (MAC) protocols have been proposed to adopt DC for achieving energy efficiency. Among them, Sensor-MAC (S-MAC) [1] belongs to the category of synchronous duty-cycled MAC protocols in which all nodes in a cluster are coordinated to follow wake-up and sleep cycles simultaneously. Currently, only few analytical models are available to evaluate the performance of the S-MAC protocol. Among them, an M/G/1 based model was proposed in [2], which requires that packet arrivals follow the Poisson process and that each node has an infinite buffer. Recently, a discrete-time Markov chain (DTMC) model was proposed in [3], supporting a more general packet arrival distribution per cycle and zero packet retransmission.

Our work extends the study presented in [3] in three different aspects. First, a DTMC was proposed in [3] to describe the evolution of the number of packets in the buffer of a node. We refer to it as the node DTMC. The solution of their model is based on the assumption that the states of nodes are mutually independent. However, in practice some degree of dependence occurs among nodes. We incorporate this dependence by introducing an additional DTMC, referred to hereafter as the system DTMC. It models the number of active nodes in the cluster. As it will be shown later, this new modeling approach substantially improves the model accuracy.

Second, we propose alternative methods to determine two important performance parameters, i.e., delay and energy

Jorge Martinez-Bauset is with the Dept. of Communications, Universitat Politècnica de València (UPV), ETSIT, Camino de Vera s/n, 46022 Valencia, Spain (email: jmartinez@upvnet.upv.es).

Lakshmikanth Guntupalli and Frank Y. Li are with the Dept. of Information and Communication Technology, University of Agder (UiA), N-4898 Grimstad, Norway (email: \{lakshmikanth.guntupalli; frank.li\}@uia.no). consumption. The new methods are shown to be more general and accurate than the ones presented in [3].

Third, instead of focusing on zero retransmission, we study scenarios where nodes are allowed to perform infinite retransmissions. S-MAC itself does not specify whether packet retransmission is allowed or not. We consider herein that the operation of S-MAC with zero retransmissions may not be appropriate for certain loss-sensitive applications due to a possibly high number of packet loss. As infinite retransmission is not a feasible mode for node operation, we investigate how many times a packet needs to be retransmitted under various traffic loads in order to achieve zero packet loss. Simulation results show that the vast majority of the packets require only one or two retransmissions, even in a high load scenario. That is, by setting the maximum retransmission counter in the nodes to two, we might achieve close to zero packet loss. This indicates that an infinite retransmission model provides a simpler modeling alternative to the more complex finite retransmission model for systems with negligible packet loss.

\section{SySTEM MODEL}

Consider a cluster of $N$ nodes consisting of sensors that are one hop away from each other. We focus on this singlecell cluster but multiple clusters together may form a larger network. The network employs S-MAC with the CSMA/CAbased RTS/CTS/DATA/ACK handshake, where backoff timers are reset at each cycle initiation. The time is partitioned into cycles, and each cycle is further divided into three parts: synchronization, data transfer and sleep. In S-MAC, a node goes to sleep until the next data (listen) period when: i) it loses the contention (hears a busy medium before its backoff timer expires); ii) it encounters an RTS collision; and iii) after successful transmission (only one packet sent per cycle).

A homogeneous channel traffic generation is assumed, i.e., the fraction of packets sent from one node to any other node is $1 /(N-1)$. For simplicity we assume a renewal arrival process, and characterize the number of packets that arrive to a node per cycle by independent and identically distributed random variables. In each cycle, packets arrive following a general distribution irrespective of where the packets were originated, i.e., internally (as a consequence of its own sensing activity) or externally (arriving from another node). Every node has a queue that can store at most $Q$ packets.

\section{A. Access to the Medium}

Consider an arbitrarily selected node as the reference node $(\mathrm{RN})$. Active nodes are those with a non-empty queue, i.e., with packets to send. The active nodes generate a random 
backoff time selected from $\{0, \ldots, W-1\}$. When the $\mathrm{RN}$ is active, it transmits a packet successfully (without collision) if the other contending nodes selected backoff times greater than the one chosen by the RN. A packet transmitted by the RN will fail (collide) when more than one contending nodes select the same backoff time as the RN, and the backoff time is the smallest among all contending nodes. When the backoff time generated by the RN is not the smallest one among those generated by the other contending nodes, two outcomes are possible: either another node is able to transmit successfully, or other nodes collide while transmitting.

Consider a cycle where the $\mathrm{RN}$ is active and denote by $k, 0 \leq k \leq N-1$, the number of nodes that are also active in the same cycle in addition to the RN. Let $P_{s, k}=\sum_{i=0}^{W-1}(1 / W)(W-1-i)^{k} / W^{k}$, $P_{s f, k}=\sum_{i=0}^{W-1}(1 / W)(W-i)^{k} / W^{k}$, and $P_{f, k}=$ $(1 / W) \sum_{i=0}^{W-1}\left[(W-i)^{k}-(W-1-i)^{k}\right] / W^{k}=1 / W$, be the probabilities that the $\mathrm{RN}$ transmits a packet successfully, that it transmits (successfully or with failure), and that it transmits with failure, respectively. $P_{s, k}$ is the probability that the RN selects one backoff value from 0 to $W-1$ and the other $k$ nodes choose a larger value. $P_{s f, k}$ and $P_{f, k}$ can be described in similar terms. Note that $P_{s f, k}-P_{s, k}=1 / W=P_{f, k}$ [5].

Conditioned on a successful or unsuccessful packet being transmitted by the RN when contending with other $k$ nodes, the average backoff times are $B T_{s, k}=$ $\left(1 / P_{s, k}\right) \sum_{i=0}^{W-1} i(1 / W)(W-1-i)^{k} / W^{k}$, or $B T_{f, k}=$ $\sum_{i=0}^{W-1} i(W-i)^{k} / W^{k}-\sum_{i=0}^{W-1} i(W-1-i)^{k} / W^{k}$.

\section{B. Node DTMC}

Here we model the evolution of the number of packets in the queue (state) of the RN over time by a DTMC. At each data period, state transition opportunities occur when: i) the $\mathrm{RN}$ is active and it transmits a packet (state can decrease); and ii) a packet is received by the RN (state can increase). Consider the homogeneous case where all the nodes behave as the RN does and also support infinite retransmissions.

Let $A_{i}$ be the probability that $i$ packets arrive to the $\mathrm{RN}$ in a cycle of length $T$. If packets arrive to a node following a Poisson process with rate $\lambda$, then $A_{i}=(\lambda T)^{i} \cdot e^{-\lambda T} / i$ ! and $\widehat{A}_{i}=1-\sum_{0}^{i-1} A_{i}$ where $\widehat{A}_{i}$ denotes the probability that at least $i$ packets arrived during $T$. Note that our model applies to any other distribution for $A_{i}$ as well. Let $\boldsymbol{P}=\left[P_{i, j}\right]$ be the transition probability matrix of the node DTMC, where $P_{i, j}$ is the probability that $j$ packets are found in the queue at cycle $m+1$, conditioned on finding $i$ packets in the queue at cycle $m$. These transition probabilities are defined by,

$$
\begin{aligned}
& P_{0, i}=A_{i}, i \leq Q-1, \quad P_{0, Q}=\widehat{A}_{Q}, \\
& P_{i, j}=p_{s} A_{j-i+1}+\left(1-p_{s}\right) A_{j-i}, \\
& \quad i=0 \ldots Q-1, j=i \ldots Q-1, \\
& P_{i, Q}=p_{s} \widehat{A}_{Q-i+1}+\left(1-p_{s}\right) \widehat{A}_{Q-i}, \quad i=1 \ldots Q, \\
& P_{i, i-1}=p_{s} A_{0}, i=1 \ldots Q, \quad P_{i, j}=0, j \leq i-2 .
\end{aligned}
$$

The solution of this DTMC can be obtained by solving the set of linear equations

$$
\boldsymbol{\pi}=\boldsymbol{\pi P}, \quad \boldsymbol{\pi} \boldsymbol{e}=1,
$$

where $\boldsymbol{\pi}$ is the stationary distribution vector and $\boldsymbol{e}$ is a column vector of ones. For the node DTMC, we adopt the common approximation that the probability of successful transmission, $p_{s}$, is independent of the state of the other nodes.

Let $M_{k}\left(\pi_{0}\right)$ be the probability that $k$ nodes, out of the $N-1$ ones other that the $\mathrm{RN}$, are active, where $\pi_{0}$ is the probability that a node is inactive. Assuming a binomial distribution for the number of active nodes [3], $M_{k}\left(\pi_{0}\right)=$ $\left(\begin{array}{c}N-1 \\ k\end{array}\right)\left(1-\pi_{0}\right)^{k} \pi_{0}^{N-1-k}$. Then,

$$
p_{s}\left(\pi_{0}\right)=\sum_{k=0}^{N-1} M_{k}\left(\pi_{0}\right) P_{s, k},
$$

is the (average) probability that the $\mathrm{RN}$ transmits a packet successfully, conditioned on the RN being active.

By solving the set of equations in $(1), \pi_{0}\left(p_{s}\right)$ can be determined for a given $p_{s}$. Then, a new $p_{s}\left(\pi_{0}\right)$ can be obtained from (2) for a given $\pi_{0}$. Denote by $p_{s}$ the solution of this fixed-point equation, i.e., the value of $p_{s}\left(\pi_{0}\right)$ at the fixedpoint. A different approach for determining $p_{s}$ is proposed in the next subsection using the system DTMC, considering that the binomial distribution assumption may not hold.

Note that the node DTMC model applies to zero retransmission as well if $p_{s}$ is replaced by $p_{s f}$ in (1). As in [3], the probability that the RN transmits a packet (successfully or with failure), $p_{s f}$, can be obtained by solving a similar fixed-point equation but with $p_{s f}\left(\pi_{0}\right)=\sum_{k=0}^{N-1} M_{k}\left(\pi_{0}\right) P_{s f, k}$.

\section{System DTMC for Infinite Retransmissions}

The system DTMC models the evolution of the number of active nodes in the cluster. Let $\boldsymbol{P}^{\prime}=\left[P_{i, j}^{\prime}\right]$ be the transition probability matrix of the DTMC that models the evolution of the number of active nodes in the system, where $P_{i, j}^{\prime}$ is the probability that $j$ nodes are found active at cycle $m+1$, conditioned on finding $i$ active nodes at cycle $m$. The transition probabilities of the system DTMC are:

$$
\begin{aligned}
& P_{0 j}^{\prime}= B_{j}(N), 0 \leq j \leq N, \\
& P_{i j}^{\prime}=\left(\widehat{S}_{i}+S_{i} \widehat{E}\right) B_{j-i}(N-i)+S_{i} E B_{j-i+1}(N-i), \\
& \quad 1 \leq i \leq N-1, i \leq j \leq N-1, \\
& P_{i N}^{\prime}=\left(\widehat{S}_{i}+S_{i} \widehat{E}\right) B_{N-i}(N-i), 1 \leq i \leq N-1, \\
& P_{N N}^{\prime}=\widehat{S}_{N}+S_{N} \widehat{E}=\left(1-S_{N} E\right), \\
& P_{i j}^{\prime}=0,2 \leq i \leq N, j<i-1, \\
& P_{i j}^{\prime}=S_{i} E B_{0}(N-i), 1 \leq i \leq N-1, j=i-1, \\
& P_{N N-1}^{\prime}=S_{N} E,
\end{aligned}
$$

where: i) $B_{j}(n)=\left(\begin{array}{c}n \\ j\end{array}\right) \widehat{A}_{1}^{j} A_{0}^{n-j}$ is the probability that $j$ out of $n$ nodes that have their queues empty receive packets in a cycle, ii) $S_{k}=k P_{s, k-1}$ and $\widehat{S}_{k}=1-S_{k}$ are the probabilities that a packet is successfully transmitted in a cycle when $k$ nodes compete, and its complementary $\widehat{S}_{k}$ is the probability that packets from two or more nodes collide, and iii) $E=$ $p_{s} A_{0} \pi_{1} / p_{s}\left(1-\pi_{0}\right)$ and $\widehat{E}=1-E$ are the probabilities that a node's queue becomes empty or remains non-empty when it transmits successfully, respectively. Note that $\pi_{0}$ and $\pi_{1}$ are the stationary probabilities that the queue of a node has ' 0 ' and ' 1 ' packet, respectively. 
The solution of the system DTMC can be obtained by solving the set of linear equations in (1) but with $\boldsymbol{P}^{\prime}$. Let $\left\{\pi_{n}^{\prime}\right\}$ be the stationary distribution of the system DTMC. Furthermore, let $\alpha_{k}^{\prime}$ be the fraction of cycles where the RN is active, together with other $k$ nodes, and $\alpha_{k}$ the probability that $k$ nodes other than the $\mathrm{RN}$ are active, conditioned on the $\mathrm{RN}$ being active. Then, $\alpha_{k}^{\prime}=\left(\begin{array}{c}N-1 \\ k\end{array}\right) \pi_{k+1}^{\prime} /\left(\begin{array}{c}N \\ k+1\end{array}\right)=(k+1) \pi_{k+1}^{\prime} / N$, $\alpha_{k}\left(\pi_{0}\right)=\alpha_{k}^{\prime} / G, G=\sum_{k=0}^{N-1} \alpha_{k}^{\prime}, \quad k=0, \ldots N-1$.

Finally, $p_{s}\left(\pi_{0}\right)$ can now be obtained as $p_{s}\left(\pi_{0}\right)=$ $\sum_{k=0}^{N-1} \alpha_{k}\left(\pi_{0}\right) P_{s, k}$. Clearly, both the system and the node are one-dimensional DTMCs and they must be solved iteratively to find $p_{s}$, the fixed-point equation solution.

\section{DELAY ANALYSIS}

Let $D$ be the average delay that a packet experiences expressed in number of time cycles, i.e., from its arrival until it is transmitted and removed from the buffer. Then, $D$ can be determined by Little's law,

$$
D=\frac{N_{a v}}{\lambda_{a}}, N_{a v}=\sum_{n=0}^{Q} n \pi_{n}, \lambda_{a}=\sum_{n=0}^{Q} b_{n} \pi_{n},
$$

where $b_{0}=\sum_{\widehat{A}_{i=0}}^{Q} i A_{i}+Q \widehat{A}_{Q+1}$ and $b_{n}=\sum_{i=0}^{Q-n} i A_{i}+$ $\left(Q-n+p_{s}\right) \widehat{A}_{Q-n+1}, n>0$. Note that $\pi_{n}$ is the stationary probability of finding $n$ packets in the queue of a node, $N_{a v}$ is the average number of packets, $\lambda_{a}$ is the average number of packets that entered the queue (accepted) per cycle, and that $b_{n}$ is the mean number of packets accepted per cycle at state $n$. Note also that the last term of $b_{n}$ is obtained after solving $\left((Q-n+1) p_{s}+(Q-n)\left(1-p_{s}\right)\right) \widehat{A}_{Q-n+1}$, where we assume that only one packet can be sent per cycle.

\section{ENERGY CONSUMPTION ANALYSIS}

Define the following constants, where $P_{t x}$ and $P_{r x}$ are the transmission and reception power,

$$
\begin{aligned}
& E_{t x s}=\left(t_{R T S}+t_{D A T A}\right) P_{t x}+\left(t_{C T S}+t_{A C K}\right) P_{r x}, \\
& E_{r x s}=\left(t_{R T S}+t_{D A T A}\right) P_{r x}+\left(t_{C T S}+t_{A C K}\right) P_{t x}, \\
& E_{t x f}=t_{R T S} P_{t x}+t_{C T S} P_{r x}, \quad E_{r x f}=t_{R T S} P_{r x} .
\end{aligned}
$$

The average energy consumed by the RN during the data transfer part of a cycle when $k+1, k \geq 2$, nodes are contending for the channel, is given by

$$
\begin{aligned}
E_{d, k+1} & =q_{1, k} P_{s, k} \cdot\left[E_{t x s}+\left(4 D_{p}+B T_{s, k}\right) P_{r x}\right] \\
& +q_{1, k} P_{f, k} \cdot\left[E_{t x f}+\left(2 D_{p}+B T_{f, k}\right) P_{r x}\right] \\
& +q_{2, k} P_{s, k} \alpha_{1} \cdot\left[E_{r x s}+\left(3 D_{p}+B T_{s, k}\right) P_{r x}\right] \\
& +q_{2, k} P_{s, k} \alpha_{2} \cdot\left[E_{r x f}+\left(D_{p}+B T_{s, k}\right) P_{r x}\right] \\
& +q_{3, k} \cdot\left[E_{r x f}+\left(D_{p}+B T_{f, k}\right) P_{r x}\right] .
\end{aligned}
$$

The terms in $E_{d, k+1}$ correspond to the energy consumed by: a packet successfully transmitted, a packet transmitted that collides, a successful packet reception, a successful packet transmitted by nodes other than the RN whose destination is not the $\mathrm{RN}$, and packets transmitted by nodes other than the RN that collide, respectively.

In (4) $D_{p}$ is the one-way propagation delay, $\alpha_{1}=$ $1 /(N-1)$ and $\alpha_{2}=(N-2) /(N-1)$. Note that $\alpha_{1}$ and $\alpha_{2}$ might depend on the routing protocol. Moreover, conditioned on finding $k+1$ nodes active, $q_{1, k}=(k+1) / N$ is the probability that the $\mathrm{RN}$ is active, $q_{2, k}=k q_{1, k}+$ $(k+1)\left(1-q_{1, k}\right)$ is the average number of active nodes other than the $\mathrm{RN}$, and $q_{3, k}=q_{1, k}\left[1-(k+1) P_{s, k}-P_{f, k}\right]+$ $\left(1-q_{1, k}\right)\left[1-(k+1) P_{s, k}\right]=1-(k+1) P_{s, k}-q_{1, k} P_{f, k}$ is the probability that at least one node different from the RN transmits a packet with failure. Note that when the RN is active (with probability $q_{1, k}$ ) then $1-(k+1) P_{s, k}-P_{f, k}$ gives the probability that it does not transmit but the other $k$ nodes collide. However, when the RN is not active (with probability $\left.1-q_{1, k}\right)$ then it is still listening and reacts to the collisions of the other $k+1$ nodes in the same way.

The expressions for $E_{d, 1}$ and $E_{d, 2}$ can be easily derived from (4), while $E_{d, 0}=E_{r x f}+\left(W+D_{p}\right) P_{r x}$. Then, the average energy consumed by the RN during the data transfer part of a cycle is given by

$$
E_{d}=\sum_{n=0}^{N} R_{n} E_{d, n}
$$

where either $R_{n}=\left(\begin{array}{c}N \\ n\end{array}\right)\left(1-\pi_{0}\right)^{n} \pi_{0}^{N-n}$ or $R_{n}=\alpha_{n}$. In addition to $E_{d}$, a node also consumes energy due to the exchange of signaling messages like SYNC. Also, the energy consumed during the sleep part of a cycle is not included, as it is application dependent. Please, refer to [3] for details.

\section{NUMERICAL RESULTS}

Let $T=T_{S D} / b$ be the duration of a cycle, $T_{S D}$ the duration of the synchronization and data transfer parts, and $b$ the duty cycle fraction. We configure $T_{S D}=30 \cdot 10^{-3} \mathrm{~s}$. Then, for a given duty cycle fraction $b$, a different cycle duration $T$ is obtained. To run S-MAC we set: backoff tick $=1$, $t_{R T S}=1.8, t_{C T S}=1.8, t_{D A T A}=17.16, t_{A C K}=1.8$, and $D_{p}=2$, where all values are in units of $10^{-4} \mathrm{~s}$. The reference configuration is defined by: $N=5, Q=10, W=128$, $b=0.5, P_{t x}=52.2 \mathrm{~mW}$ and $P_{r x}=59.1 \mathrm{~mW}$. These values are similar to the ones configured in [3].

Three load conditions are considered for the reference configuration, i.e., low load (LL), medium (ML), and high (HL), which correspond to the arrival rates of $\lambda=\{1.5,3.0,4.5\}$ packets per second, respectively. As a reference, the packet loss probabilities induced by these loads due to a buffer overflow are approximately in the order of $10^{-12}, 10^{-6}$ and $10^{-1}$ respectively. During overflow episodes, some degree of selective packet discarding must occur at the sensors to give priority to the most important information. Once such imperative information is selected, the sensors rely on a lossfree transfer across the network.

We developed a discrete-event simulation model that mimics the physical behavior of the system. The simulation results are therefore completely independent from those obtained by the analytical model. We evaluate first the impact of configuring the nodes with zero retransmissions as presented in [3], versus with infinite retransmissions as proposed in this study.

In the zero retransmission mode, packet loss due to collisions in the channel obtained by simulation for the three load levels are: $\{0.435 \%, 1.81 \%, 3.92 \%\}$, respectively. When 
TABLE I

VALUeS AND PERCENTUAL RELATIVE ERRORS FOR $\pi_{0}$

\begin{tabular}{cccc}
\hline & $\pi_{0}$ & M2_err & M1_err \\
\cline { 2 - 4 } LL & 0.88 & 0.03 & 0.46 \\
ML & 0.51 & 11.76 & 23.32 \\
HL & 0.008 & 1.40 & 1.41 \\
\hline
\end{tabular}

the infinite retransmission mode is employed, we noted that more than $99.99 \%$ of the packets are transmitted after 1 or 2 retransmissions, even in the worst case (in HL). We also determined by simulation the delay and energy consumption when nodes are configured with a finite number of retransmissions. As expected, we observed that the delay and energy consumption when the maximum number of retransmissions was set to 3 or more remain practically identical, and equal to the values obtained for infinite retransmissions. This confirms that an infinite retransmission model provides a much simpler, yet accurate, alternative to a finite retransmission model for systems with negligible packet loss.

Let us now evaluate the node DTMC model in isolation versus the hybrid node and system DTMC model. Denote by model M1 the model that employs the node DTMC alone using the binomial distribution to find $p_{s}$, as proposed in [3]. The model that combines the node DTMC together with the system DTMC to find $p_{s}$, as proposed in this letter, is referred to as M2. In both models nodes have retransmissions enabled. Based on the reference configuration and the above mentioned three load levels, we compare the accuracy of these two models using the relative error $|x-y| / y$ as a measure, where $x$ is the value obtained by the corresponding analytical model and $y$ is the value obtained by simulation.

Table I presents the absolute values $\left(\pi_{0}\right)$ and percentual relative errors (M2_err and M1_err) obtained for $\pi_{0}$. Clearly, the relative error achieved by M1 is much larger, particularly at ML. This is likely due to the fact that the independence assumption of the state of the nodes in the network does not hold. Using M2, the relative error for $\pi_{0}$ is substantially reduced, especially at LL and ML. Note that at the limit of the LL and HL regimes, the distribution of active nodes would be: i) $\pi_{0}^{\prime} \cong 1$ and $\pi_{i}^{\prime} \cong 0, i \geq 1$; ii) $\pi_{N}^{\prime} \cong 1$ and $\pi_{i}^{\prime} \cong 0, i \leq N-1$, respectively. Then, in these regimes, when a node accesses the channel it always finds the same number of contenders. However, in the ML scenario finding a good distribution of active nodes is crucial to determine $p_{s}$, and therefore to solve the node DTMC accurately.

Next, we compare the average packet delay for the reference configuration but with $Q=5$, in order to highlight the differences with the delay model proposed in [3]. Columns $D_{\text {sim }}$ and $E_{d, s i m}$ in Tab. II show absolute values obtained from simulation for delay in cycles (units of $60 \mathrm{~ms}$ ) and energy consumption in units of $10^{-4} \mathrm{~J}$. Columns $D, D^{*}, E_{d}$ and $E_{d}^{*}$ show relative errors when $\left\{\pi_{n}\right\}$ is obtained by M2. Column $D$ shows the relative errors obtained using Little's law, as proposed in Sec. III. Column $D^{*}$ shows the relative errors obtained when expression (15) in [3] is adopted. As observed, the errors in $D^{*}$ are larger than those in $D$. This is likely due to the fact that expression (15) in [3] is implicitly based on the
TABLE II

VAlues AND PERCENTUAL RELATIVE ERRORS FOR M2

\begin{tabular}{ccccccc}
\hline & $D_{\text {sim }}$ & $D$ & $D^{*}$ & $E_{d, s i m}$ & $E_{d}$ & $E_{d}^{*}$ \\
\cline { 2 - 7 } $\mathrm{LL}$ & 1.42 & 0.92 & 2.15 & 5.46 & 0.20 & 4.94 \\
$\mathrm{ML}$ & 4.68 & 20.23 & 26.78 & 3.14 & 4.63 & 14.56 \\
$\mathrm{HL}$ & 17.0 & 0.42 & 12.34 & 1.85 & 0.006 & 6.19 \\
\hline
\end{tabular}

application of the arrivals see time averages (ASTA) property. However, ASTA does not hold in this system [4].

Furthermore, using also M2 and the reference configuration, we analyze the energy consumed by the $\mathrm{RN}$ during the data transfer part of a cycle. Column $E_{d}$ in Tab. II lists the relative errors obtained by expression (5) proposed in Sec. IV. Column $E_{d}^{*}$ shows the relative errors obtained by the energy model in [3]. As observed, the errors in $E_{d}^{*}$ are substantially larger than those in $E_{d}$ for all traffic load levels. This discrepancy is likely due to two facts. First, expression (4) above and expression (37) in [3] have different terms. Second, expressions for $B T_{s, k}$ and $B T_{f, k}$ derived in this letter are different from those in [3].

To further improve the accuracy, we combine both system and node DTMCs to define a two dimensional DTMC. A state in the new DTMC is represented by $(i, m)$, where $i$ is the number of packets in the queue of the RN, and $m$ is the number of active nodes in the system. With this new model, the relative errors obtained at ML for $\pi_{0}$, delay and energy consumption are $3.20 \%, 6.05 \%$ and $1.85 \%$, respectively. The relative errors obtained under LL and HL are also lower than the ones displayed in Tab. II. Details for this integrated model can be found in [5].

\section{Conclusions}

We developed an analytical model for S-MAC operating in an infinite retransmission scenario, which involves the interaction of two one-dimensional DTMCs that model the evolution of the number of packets in the queue and the number of active nodes in a cluster, respectively. The proposed model is verified by extensive discrete-event based simulations under various traffic load conditions, which show accurate results. We also proposed alternative methods to determine delay and energy consumption, which are more general and accurate than those proposed previously.

\section{REFERENCES}

[1] W. Ye, J. Heidemann, and D. Estrin, "Medium access control with coordinated adaptive sleeping for wireless sensor networks", IEEE/ACM Transactions on Networking, vol. 13, no. 3, June 2004.

[2] Y. Zhang, C. He and L. Jiang, "Performance analysis of S-MAC protocol under unsaturated conditions", IEEE Communications Letters, vol. 12, no. 3, March 2008.

[3] O.Yang and W. B. Heinzelman, "Modeling and performance analysis for duty-cycled MAC protocols with applications to S-MAC and XMAC," IEEE Trans. on Mobile Computing, vol. 11, no. 6, pp. 905-921, June 2012.

[4] B. Melamed and D. Yao, "The ASTA property," Advances in Queueing: Theory, Methods and Open Problems, pp. 195-224, 1995.

[5] J. Martinez-Bauset, L. Guntupalli, and F.Y. Li,, "Modeling and Performance Evaluation of Synchronous Duty-Cycled MAC Protocols". http://personales.upv.es/ jmartine/SMAC_2D.pdf 